\title{
AS NOVAS DCNS E OS CURSOS DE ENGENHARIA DO INSTITUTO FEDERAL DE EDUCAÇÃO, CIÊNCIA E TECNOLOGIA DO CEARÁ
}

Bruno Noronha Rodrigues - bruno.noronha@ifce.edu.br Instituto Federal de Educação, Ciência e Tecnologia do Ceará, Campus Crateús Avenida Dr. Geraldo Barbosa Marques, 567 - Venancio 63708-260-Crateús - Ceará

Jorge Vleberton Bessa de Andrade-jorgevleberton@unifei.edu.br Instituto de Sistemas Elétricos e Energia, Universidade Federal de Itajubá Avenida BPS, 1303 - Pinheirinho 37500-903 - Itajubá - Minas Gerais

Resumo: As Novas Diretrizes Curriculares Nacionais (DCNs) dos Cursos de Graduação em Engenharia foram aprovadas pelo Ministério da Educação, por meio da Resolução CNE/CES $n^{\circ} 2$, em abril de 2019. O motivo para a mudança se deve as demandas do mundo do trabalho contemporâneo e o perfil dos jovens discentes. Esse trabalho tem o intuito de avaliar a atual situação dos Projetos Pedagógicos e as possibilidades da inserção das novas DCNs nos cursos de engenharia da área de Construção Civil do Instituto Federal de Educação, Ciência e Tecnologia do Ceará (IFCE). Os procedimentos metodológicos seguiram os passos para estudos de casos múltiplos com o desenvolvimento de um checklist (com quatro categorias e sessenta e três itens), seguido da aplicação em quatro Projetos Pedagógicos de cursos de engenharia e análise e cruzamento dos resultados. Após o diagnóstico situacional percebeuse que os cursos cumprem mais de $85 \%$ dos itens previstos nas novas diretrizes, porém é necessário a implementação de alguns pontos importantes como o uso de metodologias ativas, o incentivo a participação de empresas juniores e um programa de formação $e$ desenvolvimento do corpo docente.

Palavras-chave: DCNs. Projetos Pedagógicos de Cursos. Engenharias. IFCE.

\section{INTRODUÇÃO}

De acordo com a Organisation for Economic Co-operation and Development (OECD) (2019), alguns setores ainda lutam para encontrar trabalhadores qualificados que necessitam. Embora o de Engenharia, Fabricação e Construção seja comumente associado aos melhores resultados do mercado de trabalho, apenas $14 \%$ dos graduados, em todo o mundo, se formaram na área em 2017. O Brasil ocupa a $44^{\circ}$ posição, de um total de 46 países membros, no percentual de graduados na área em relação a todas as outras.

Esta situação, segundo Watanabe et al. (2019), pode ser entendida como sendo resultante da conjunção de dois principais fatores: a baixa procura por cursos das áreas de Ciência, Tecnologia, Engenharia e Matemática (STEM, do inglês Science, Technology, Engineering, and Mathematics) e os índices elevados de retenção e evasão de estudantes destas carreiras.

Rask (2010) explica que a baixa procura por cursos das ciências exatas pelos estudantes brasileiros, deve-se pelas dificuldades encontradas nas disciplinas de matemática, física e química no ensino básico, muitas crianças e adolescentes estabelecem uma espécie de bloqueio para aprender e dominar este conteúdo. Uma consequência desse bloqueio diz respeito ao crescente desinteresse dos jovens por programação e pela área de Tecnologia da Informação (TI). Além disso, esse bloqueio acaba por tornar os cursos da área de humanas 
mais atrativos do que os cursos de exatas, o que demanda estratégias para torná-los mais interessantes (MENESES et al., 2015).

O ingresso de estudantes sem prévio conhecimento do que é abordado e oferecido em um curso de engenharia, em especial nos primeiros anos da formação, pode ser considerado um dos motivos de evasão. Esses problemas possuem efeitos danosos perceptíveis, principalmente para a sociedade (SILVA FILHO et al., 2007). A evasão aumenta o custo unitário da formação dos estudantes, pois a mesma estrutura acadêmica e recursos humanos que deveriam atender um dado grupo inicial são destinados a um quantitativo menor de indivíduos. A retenção também apresenta seus impactos negativos, ao não permitir que profissionais de nível superior venham a atuar nas suas respectivas áreas do conhecimento no prazo inicialmente previsto. Além disso, esses estudantes retidos também podem, em algum momento, evadir-se (CAMPELO; LINS, 2008).

Silva e Cecílio (2007) relatam que alguns estudantes não conseguem relacionar os interesses comuns entre aquilo que têm de aprender e o que precisarão saber para a atuação profissional futura. Essa diferença é importante, uma vez que os conteúdos das disciplinas básicas, como a Matemática, a Física, entre outras, são necessários para subsidiar as disciplinas profissionalizantes, as quais são de mais fácil identificação quanto à aplicação direta na profissão. Para Carvalho et al. (2018) a pouca compreensão da aplicabilidade, na prática profissional, origina desmotivação.

Discentes desmotivados apresentam alto índice de distração, baixa participação nas aulas, distanciando-se de um processo mais concreto de aprendizagem (CAVENAGUI; BZUNECK, 2009). Em contrapartida, um estudante motivado mostra-se proativo, esforçado e entusiasmado com a possibilidade de aprender e se qualificar (BZUNECK; BORUCHOVITCH, 2003).

$\mathrm{Na}$ outra ponta, temos, o mercado de trabalho globalizado, altamente conectado que tem exigido que os futuros engenheiros sejam mais do que tecnicamente qualificados. Os profissionais de engenharia deste século devem ser também flexíveis, capazes de solucionar problemas e devem pensar crítica, criativa e dinamicamente. Assim sendo, a ideia de priorização do conteúdo técnico deve ser abandonada em detrimento da necessidade de desenvolvimento das novas competências e habilidades do século XXI, inerentes aos profissionais da área de engenharia. As Habilidades do Século XXI são definidas como os conhecimentos e procedimentos que podem ser transferidos ou aplicados em novas situações (NATIONAL RESEARCH COUNCIL, 2012).

Frente a essa problemática, as Diretrizes Curriculares Nacionais do Curso de Graduação em Engenharia (DCNs de Engenharia) foram atualizadas em abril de 2019 pelo Conselho Nacional de Educação (CNE) do Ministério da Educação (MEC) (BRASIL, 2019). Após um processo de ampla discussão com entidades de representação acadêmica, industrial e profissional, tais como a Associação Brasileira de Educação em Engenharia (Abenge), a Confederação Nacional da Indústria (CNI) e o Conselho Federal de Engenharia e Agronomia (Confea) (WATANABE et al. (2019). Como resultado, as novas DCNs de Engenharia possibilitaram a flexibilização dos Projetos Pedagógicos dos Cursos (PPCs) e mudaram a concepção de formação por meio de conteúdos para uma formação por competências. Além disso, foram evidenciadas preocupações diversas como o estímulo às atividades práticas para o exercício da criatividade e do espírito de inovação, a adoção de metodologias de aprendizagem ativa, a diversificação dos instrumentos de avaliação, o acolhimento dos ingressantes e a formação e valorização da atividade docente, dentre outros. A presente pesquisa objetiva avaliar a atual situação dos PPCs e as possibilidades da inserção das novas DCNs nos cursos de engenharia da área de Construção Civil do Instituto Federal de Educação, Ciência e Tecnologia do Ceará (IFCE). 


\section{CURSOS DE ENGENHARIA NO IFCE E SEUS PROJETOS PEDAGÓGICOS}

O Instituto Federal de Educação, Ciência e Tecnologia do Ceará (IFCE), cuja reitoria é sediada em Fortaleza, foi criado nos termos da Lei. $\mathrm{N}^{\circ} 11.892$, de 29 de dezembro de 2008, mediante a integração do Centro Federal de Educação Tecnológica do Ceará com as Escolas Agrotécnicas Federais de Crato e de Iguatu, vinculado ao Ministério da Educação, é uma autarquia de natureza jurídica, detentora de autonomia administrativa, patrimonial, financeira, didático-pedagógica e disciplinar. Para efeito da incidência das disposições que regem a regulação, avaliação e supervisão da instituição e dos cursos de educação superior, o IFCE é equiparado às universidades federais (IFCE, 2020).

As raízes da instituição remontam ao começo do século $\mathrm{XX}$, quando o então presidente Nilo Peçanha, pelo Decreto $\mathrm{n}^{\mathrm{o}}$ 7566, de 23 de setembro de 1909, instituiu a Escola de Aprendizes Artífices. Ao longo de um século de existência, a instituição teve sua denominação alterada, primeiro para Liceu Industrial do Ceará, em 1941; depois para Escola Técnica Federal do Ceará, em 1968. No ano de 1994, a escola passou a chamar-se Centro Federal de Educação Profissional e Tecnológica do Ceará (Cefet/CE), ocasião em que o ensino foi estendido ao nível superior e suas ações acadêmicas, acrescidas das atividades de pesquisa e extensão. O IFCE tem forte atuação nas áreas da pesquisa e da extensão, com foco especial nas linhas atinentes às áreas técnica e tecnológica.

Dados de fevereiro de 2020 mostram que o instituto conta com 3.712 servidores entre professores e técnicos administrativos e 31.729 alunos matriculados, distribuídos por 32 campi, em todas as regiões do Ceará, e em 289 cursos distintos, sendo eles de nível básico, técnico, graduação e pós-graduação. Os cursos de graduação em engenharia, por sua vez, somam 14, em 6 campi diferentes: Engenharia Ambiental (Juazeiro do Norte); Engenharia Ambiental e Sanitária (Maracanaú e Quixadá); Engenharia Civil (Fortaleza, Morada Nova e Quixadá); Engenharia de Aquicultura (Morada Nova e Aracati); Engenharia de Computação (Fortaleza); Engenharia de Controle e Automação (Maracanaú); Engenharia de Mecatrônica (Fortaleza); Engenharia de Produção Civil (Quixadá); Engenharia de Telecomunicações (Fortaleza); e Engenharia Mecânica (Maracanaú) (IFCE, 2020a).

As novas DCNs de Engenharia são os principais elementos norteadores para os processos de criação ou reformulação de cursos de graduação em engenharia, contudo, outras normativas, internas às Instituições de Ensino Supeior (IES) ou estabelecidas pelo MEC devem ser consideradas nesses processos. No caso do IFCE, tem-se o Manual de Elaboração de Projetos Pedagógicos dos Cursos. O atual, aprovado em 2017, serve como normatizador para elaboração de Projeto Pedagógico de Curso (PPC) de cursos técnicos e de graduação, foi pensado para ser uma ferramenta estratégica de referência tanto na elaboração, no planejamento, na avaliação, como na atualização dos PPCs técnicos, em suas diversas formas (integrados, subsequentes e concomitantes), e de graduação, nas modalidades a distância e presencial, ofertados pelos campi. Foi estruturado em tópicos, com bibliografia e com a fundamentação legal necessária.

Consoante o manual, o PPC é o documento que apresenta os pressupostos teóricos e metodológicos que fundamentam um curso, assim como sua estrutura e funcionamento. É um documento de referência das instituições de ensino quando se trata do retrato de um curso, pois, dos aspectos citados, apresenta o percurso formativo do estudante por meio de uma organização curricular que deve estabelecer estratégias formativas coerentes com as áreas de conhecimento contempladas (IFCE, 2017).

A versão atual das DCNs de Engenharia, estabelecidas pela Resolução CNE/CES N ${ }^{\circ}$ 2/2019 (BRASIL, 2019) é estruturada por 18 artigos distribuídos em 6 capítulos e substitui a versão anterior de 2002 (BRASIL, 2002). Um trabalho comparativo, com o objetivo de 
identificar e sistematizar as principais mudanças e inovações propostas nas novas diretrizes, foi realizado por Oliveira (2019), os resultados da pesquisa foram sintetizados e organizados em um quadro por Watanabe et al. (2019).

Quadro 1 - Principais mudanças e inovações das novas DCNs de engenharia

\begin{tabular}{|c|c|}
\hline Tó & \\
\hline $\begin{array}{l}\text { Perfil do } \\
\text { (Art. }\end{array}$ & $\begin{array}{l}\text { Perfil consonante com as atuais necessidades de formação em Engenharia e } \\
\text { compreende as seguintes características: visão holística e humanista, formação técnica } \\
\text { forte; aptidão para a pesquisa e desenvolvimento de forma inovadora e empreendedora; } \\
\text { atenção às necessidades do usuário; visão multidisciplinar e transdisciplinar; } \\
\text { preocupação com aspectos globais; e comprometimento com a responsabilidade social } \\
\text { e o desenvolvimento sustentável. }\end{array}$ \\
\hline $\begin{array}{l}\text { Competências do } \\
\left.\text { egresso (Art. } 4^{\circ}\right)\end{array}$ & $\begin{array}{l}\text { Além de competências técnicas e de gestão, abrangem outras mais gerais associadas à } \\
\text { comunicação, trabalho em equipe, ética profissional e aprendizagem autônoma. Os } \\
\text { cursos devem ser organizados com foco no desenvolvimento de competências } \\
\text { explicitadas nas novas DCNs. }\end{array}$ \\
\hline $\begin{array}{l}\text { Áreas de atuação } \\
\qquad\left(\text { Art. } 5^{\circ}\right)\end{array}$ & $\begin{array}{l}\text { Dependendo do perfil e das competências dos egressos, previstas no PPC, os } \\
\text { engenheiros podem atuar nos contextos de projeto e inovação e/ou de empreendimento } \\
\text { e gestão, e/ou ainda, na formação acadêmica e atualização profissional. }\end{array}$ \\
\hline $\begin{array}{l}\text { Estrutur } \\
\qquad \text { (Ar }\end{array}$ & $\begin{array}{l}\text { Os itens principais que devem compor o PPC são explicitados nas novas DCNs de } \\
\text { Engenharia, reforçando sua importância para a estruturação do curso com base em } \\
\text { atividades de aprendizagem que articulem teoria, prática, projeto, aprendizagem ativa, } \\
\text { contexto de aplicação, pesquisa, extensão e ambiente profissional. }\end{array}$ \\
\hline $\begin{array}{l}\text { Acolhi } \\
\text { niveli } \\
\quad(\mathrm{Ar}\end{array}$ & $\begin{array}{l}\text { onsiderando a heterogeneidade dos ingressantes, tanto em termos de conhecimentos } \\
\text { ásicos quanto em aspectos psicopedagógicos, devem ser previstos sistemas de apoio, } \\
\text { sando melhorar as condições permanência e a diminuição de retenção e evasão dos } \\
\text { gressantes. }\end{array}$ \\
\hline $\begin{array}{r}\mathrm{Ca} \\
\text { horária/ } \\
\text { integr } \\
\quad(\mathrm{Ar}\end{array}$ & $\begin{array}{l}\text { Explicitados nas novas DCNs ao remeter à Resolução CNE/CES No } 2 \text { de } 2007 . \\
\text { Indicam ainda os diferentes tipos de atividades acadêmicas curriculares e a } \\
\text { possibilidade de articulação com programas de pós-graduação stricto sensu. }\end{array}$ \\
\hline $\begin{array}{l}\text { Conte } \\
\text { PPC }\end{array}$ & $\begin{array}{l}\text { eúdos básicos obrigatórios, possibilitando a definição dos } \\
\text { rofissionais de forma mais flexível, mas destacando a } \\
\text { vidades práticas e de laboratório. }\end{array}$ \\
\hline $\begin{array}{r}\text { Ati } \\
\text { Compl } \\
(\mathrm{A}\end{array}$ & $\begin{array}{l}\text { Devem contribuir efetivamente para o desenvolvimento das competências previstas no } \\
\text { PPC. }\end{array}$ \\
\hline $\begin{array}{r}\text { Estági } \\
\text { obr } \\
(A\end{array}$ & da Engenharia \\
\hline $\begin{array}{l}\text { Projeto Final de } \\
\text { Curso - PFC (Art. } \\
\text { 12) }\end{array}$ & $\begin{array}{l}\text { A adoção da nova terminologia - Projeto, em substituição a - Trabalho, remete à } \\
\text { atividade primordial de um engenheiro. O PFC ser desenvolvido de forma individual } \\
\text { ou em equipe e deve demonstrar a capacidade de articulação das competências } \\
\text { inerentes à formação do engenheiro. }\end{array}$ \\
\hline $\begin{array}{l}\text { Avalic } \\
\text { ativ } \\
\text { (Ar }\end{array}$ & $\begin{array}{l}\text { avaliação da aprendizagem e das competências dos estudantes deve ser organizada } \\
\text { mo parte indissociável das atividades acadêmicas, e o processo avaliativo deve ser } \\
\text { jersificado e adequado às diferentes atividades do curso. }\end{array}$ \\
\hline $\begin{array}{r}\text { Corpo } \\
(\mathrm{Ar}\end{array}$ & $\begin{array}{l}\text { O corpo docente deverá estar alinhado com o previsto no PPC e, para tanto, indica-se a } \\
\text { necessidade de formação pedagógica específica; além disso, as IES deverão definir } \\
\text { indicadores de avaliação e valorização do trabalho docente nas atividades de } \\
\text { graduação. }\end{array}$ \\
\hline $\begin{array}{l}\text { Implantação e } \\
\text { Desenvolvimento } \\
\text { (Art. 15) }\end{array}$ & $\begin{array}{l}\text { Além dos processos de avaliação e regulação conduzidos pelo MEC, a implantação e o } \\
\text { desenvolvimento das novas DCNs de Engenharia devem ser acompanhados, } \\
\text { monitorados e avaliados pelas próprias IES. }\end{array}$ \\
\hline Prazo para & DCNs devem ser implementadas pelos c \\
\hline
\end{tabular}




\begin{tabular}{|c|l|}
\hline $\begin{array}{c}\text { Implementação } \\
\text { (Art. 16) }\end{array}$ & $\begin{array}{l}3 \text { anos, a partir de 24/4/2019, de forma gradual ou imediatamente, com a devida } \\
\text { anuência dos alunos. }\end{array}$ \\
\hline $\begin{array}{c}\text { Instrumentos de } \\
\text { avaliação de } \\
\text { curso (Art. 17) }\end{array}$ & $\begin{array}{l}\text { Indica-se a necessidade de adequação dos instrumentos de avaliação de curso em } \\
\text { função das alterações das novas DCNs de Engenharia. }\end{array}$ \\
\hline
\end{tabular}

Fonte: Adaptado de Watanabe et al. (2019) e Oliveira (2019).

\section{METODOLOGIA}

A metodologia deste trabalho baseia-se na abordagem de estudos de casos múltiplos descrito por Yin (2015), composta por três etapas principais: definição e planejamento; preparação, coleta e análise; e conclusões. A utilização dessa estratégia foi escolhida por se enquadrar na forma da questão de pesquisa (como e por que), da não exigência de controle sobre os eventos comportamentais e do foco em acontecimentos contemporâneos. A Figura 1, mostra o fluxograma da pesquisa, com todas as etapas e sub etapas da pesquisa.

Figura 1 - Metodologia da pesquisa.

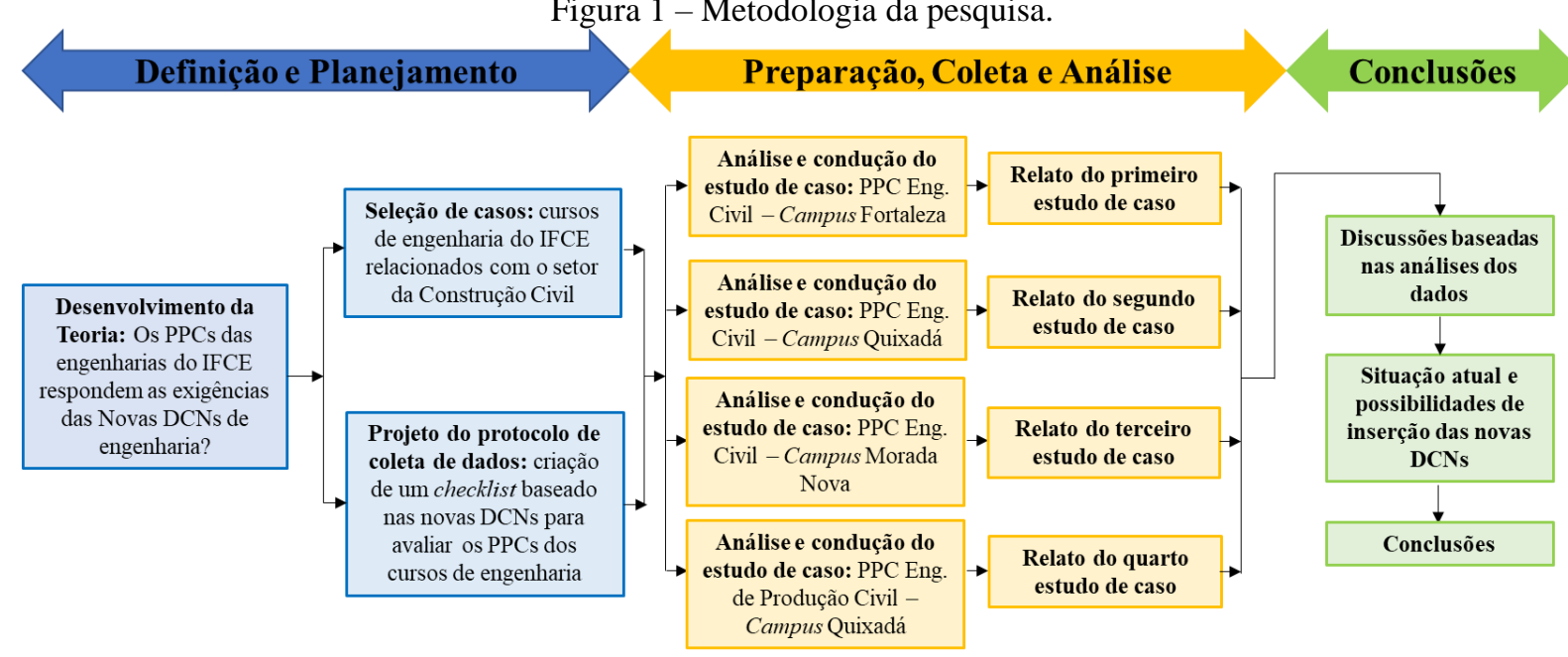

Fonte: Adaptado de Yin (2015).

$\mathrm{Na}$ primeira etapa, com base no objetivo geral do estudo, foi desenvolvida a teoria investigativa sobre a atual situação dos PPCs dos cursos de engenharia do IFCE e como eles cumprem ou não as exigências estabelecidas nas novas DCNs. Na seleção de casos para estudo, optou-se por restringir a pesquisa aos cursos relacionados, diretamente, com o setor da Construção Civil. Dos 14 cursos de engenharia, 4 se inserem no contexto: Engenharia Civil nos campi de Fortaleza, Quixadá e Morada Nova; e Engenharia de Produção Civil no campus de Quixadá.

Ainda na etapa inicial, para o protocolo de coleta de dados, foi criada uma lista de verificação (Checklist), com as exigências da Resolução CNE/CES No 2/2019. O instrumento foi dividido em 4 categorias: (a) organização do curso; (b) perfil e competências do egresso; (c) avaliação das atividades; e (d) corpo docente. As categorias foram divididas em 63 itens. $\mathrm{O}$ preenchimento dos itens foi realizado por dois pesquisadores que avaliaram se o quesito era contemplado ou não no PPC estudado e faziam observações acerca das divergências e ausências encontradas.

A segunda etapa foi constituída pela análise dos quatro PPCs de maneira individual, sendo cada um considerado um estudo de caso, então foi realizado relatórios individuais com 
os resultados. Professores e coordenadores dos cursos foram consultados quando os pesquisadores tinham alguma dúvida em relação ao PPC.

A última etapa foi estabelecida com as discussões acerca dos relatórios individuais, com a situação atual dos PPCs, conclusões e possibilidades de inserção das novas DCNs de engenharia.

\section{RESULTADOS E DISCUSSÕES}

Os resultados aqui apresentados baseiam-se nos estudos de casos individuais dos PPCs após a aplicação do checklist. $\mathrm{O}$ acesso aos documentos foi realizado por meio do site oficial de cada curso. Além das informações sobre as Novas DCNs, contidas nos 63 itens avaliados, outras mais gerais foram extraídas, como resumidas no Quadro 2.

Quadro 2 - Informações sobre os cursos analisados

\begin{tabular}{|c|c|c|c|c|}
\hline & $\begin{array}{c}\text { Eng. Civil / } \\
\text { Fortaleza }\end{array}$ & $\begin{array}{c}\text { Eng. Civil / } \\
\text { Quixadá }\end{array}$ & $\begin{array}{c}\text { Eng. Civil / } \\
\text { Morada Nova }\end{array}$ & $\begin{array}{c}\text { Eng. de Produção } \\
\text { Civil / Quixadá }\end{array}$ \\
\hline Inauguração do curso & 2012 & 2018 & 2016 & 2015 \\
\hline Última atualização do PPC & 2019 & 2017 & 2015 & 2015 \\
\hline $\mathrm{N}^{\circ}$ de vagas semestrais & 30 & 35 & 30 & 30 \\
\hline $\mathrm{N}^{\circ}$ de alunos matriculados & 240 & 134 & 149 & 208 \\
\hline $\mathrm{N}^{\circ}$ de formandos & 73 & 0 & 0 & 0 \\
\hline Carga horária total & 4440 & 4360 & 4360 & 4000 \\
\hline
\end{tabular}

Fonte: Autoria própria (2019).

Como pode ser observado os cursos de engenharia no IFCE foram criados recentemente, sendo que dos quatro avaliados, apenas a Engenharia Civil do campus Fortaleza possui egressos, além de ser o único em que ocorreu atualização do PPC após a sua instauração. As vagas ofertadas para ingressos são disponibilizadas semestralmente, com exceção da Engenharia Civil do campus Morada Nova que oferece vagas apenas uma vez por ano. As cargas horárias para os cursos cumprem o estabelecido na Resolução CNE/CES nº 2, de 18 de junho de 2007, citada como referência nas Novas DCNs, que é de no mínimo 3600 horas para cursos de engenharia.

$\mathrm{Na}$ avaliação geral das 4 categorias (organização do curso; perfil e competências do egresso; avaliação das atividades; e corpo docente) do checklist, os cursos apresentaram resultados acima de $85,00 \%$, ou seja, mais de 53 itens já são contemplados nos atuais PPCs, como mostrado no Gráfico 1. Todos os conteúdos básicos necessários, definições do perfil do egresso e os itens da categoria "avaliação das atividades" estão presentes nos documentos. 
Gráfico 1 - Itens das DCNs atendidos pelos PPCs



Fonte: Autoria própria (2019).

Entre os requisitos não contemplados, o curso de Engenharia de Produção Civil apresenta melhor resultado, com apenas 4 itens não atendendo as determinações da Resolução de 2019. Seguido da Engenharia Civil campus Fortaleza com 6 itens e as dos campi de Morada Nova e Quixadá com 7 itens. Além desses requisitos, outros dois, foram parcialmente atendidos. O item "Projeto final do curso" não foi tratado da maneira apropriada por nenhum PPC, já que a atualização defende a mudança do termo Trabalho por Projeto, sendo a atividade primordial do engenheiro e correspondendo na prática a vários tipos de atividades que podem ser realizadas para comprovação final da aprendizagem dos discentes. $\mathrm{O}$ outro item, atendido parcialmente por dois PPCs, foi "Formular e conceber soluções desejáveis de engenharia, analisando e compreendendo os usuários dessas soluções e seu contexto", por não refletir exatamente a importância da questão do contexto na resolução de problemas dos usuários.

As deficiências encontradas nos PPCs, frente as Novas DCNs, são semelhantes na maioria dos itens. Na categoria "Organização do curso", destacam-se o "Estímulo ao uso de metodologias ativas" e o "Estímulo a competições acadêmicas" que não aparecem em nenhum PPC. A aprendizagem ativa busca aproximar estudantes da realidade enfrentada pelas empresas por meio do desenvolvimento do pensamento crítico e do uso de habilidades físicas e cognitivas. Cada vez mais os cursos de engenharia caminham para mudança nos processos de ensino-aprendizagem, uma possibilidade bastante explorada atualmente é a metodologia de Aprendizagem Baseada em Problemas (ABP), onde os discentes adquirem conhecimento a partir de informações e dados coletados por eles, em um ambiente exploratório.

Ainda na mesma categoria, os itens de estímulos "ao desenvolvimento de protótipos", "a participação em empresas juniores" e "acompanhamento do egresso", são contemplados apenas na Engenharia de Produção Civil. O caso da execução de protótipos destaca-se, principalmente, na Engenhara de Produto, área específica da Produção, porém, as DCNs destacam a execução de atividades práticas como de grande importância para o ensino de engenharia, ou seja, a engenharia civil também deve estimular essa abordagem.

Criar ferramentas de acompanhamento do egresso é necessário para qualquer curso, visto que o concludente é o principal produto no processo de formação e seus resultados auxiliam o melhoramento constante do PPC. A Assistência Social, dentro do serviço de atendimento ao discente, é o setor responsável por esse processo, segundo o PPC da Engenharia de Produção Civil. A participação em empresas juniores, aparece no mesmo projeto pedagógico como uma possível atividade de gestão, para as horas complementares dos discentes. O principal 
objetivo desse tipo de dinâmica é a complementação dos conteúdos que são vistos em sala de aula e o contato com profissionais da área antes mesmo dos alunos estarem no mercado.

As Novas DCNs determinam que o corpo docente deve estar alinhando com o previsto no PPC, deve passar por formação pedagógica e indicadores de avaliação para valorização do trabalho devem ser criados. Porém, nota-se que essa categoria é tratada de maneira bem precária nos PPCs analisados. O item "Programa de formação e desenvolvimento do corpo docente" não aparece de maneira clara em nenhum documento e o item "Definição de indicadores de avaliação e valorização do trabalho docente" aparece somente em dois. O PPC da Engenharia Civil/Fortaleza trata da temática no tópico Avaliação de Aprendizagem destacando a aplicação de questionário de avaliação do trabalho docente pelos alunos como iniciativa da Coordenação Técnico Pedagógica (CTP) que, por meio de constante processo de avaliação, aprimora o ensino por parte do corpo docente.

\section{CONCLUSÕES}

As novas DCNs de Engenharia trouxeram mudanças profundas na concepção de um curso de graduação e na implementação de temáticas relacionadas ao acolhimento e nivelamento de discentes ingressantes. Além disso o trabalho docente passou a ser repensado, com a necessidade de uma valorização e formação pedagógica contínua. Ao avaliar os quatro cursos de engenharia da área de construção civil do IFCE percebeu-se que várias normativas internas e externas, incluindo mais de $85 \%$ dos itens das novas DCNs, já são atendidas. Por outro lado, outros itens demandam bastante reflexão para incorporação nos PPCs.

É importante ressaltar que a pesquisa utilizou os documentos oficiais de pressupostos teóricos e metodológicos que fundamentam cada um dos cursos e em alguns momentos professores e coordenadores foram consultados, não avaliando a prática das atividades ali propostas. Partiu-se da hipótese do seguimento integral do PPC.

Considerando o prazo de implementação do exposto na Resolução CNE/CES n 2, que são três anos, os cursos do IFCE apresentam-se flexíveis as alterações, visto que poucos itens não foram atendidos e outros necessitam apenas de reformulação. Toda mudança nesse sentido gera apreensão inicial e desafios ao trabalho de coordenadores, docentes e equipes pedagógicas. Porém, a engenharia é um espaço de sucessivas propostas inovadoras, sendo assim, a proposição de um novo projeto pedagógico, alinhado com as necessidades atuais, é de extrema importância.

\section{REFERÊNCIAS}

BRASIL. Ministério da Educação. Conselho Nacional de Educação. Câmara de Educação Superior. Resolução $\mathbf{n}^{\mathbf{0}} \mathbf{1 1}$, de 11 de março de 2002. Institui as Diretrizes Curriculares Nacionais do Curso de Graduação em Engenharia. Brasília: Ministério da Educação, 2002. Disponível em: http://portal.mec.gov.br/cne/arquivos/pdf/ CES112002.pdf . Acesso em: 28 mai. 2020.

BRASIL. Ministério da Educação. Conselho Nacional de Educação. Câmara de Educação Superior. Resolução n⿳0 2, de 24 de abril de 2019. Institui as Diretrizes Curriculares Nacionais do Curso de Graduação em Engenharia. 2019. Brasília: Ministério da Educação, 2019. Disponível em:

http://portal.mec.gov.br/index.php?option=com_docman\&view=download\&alias $=112681$ rces002-19\&category_slug=abril-2019-pdf\&Itemid=30192 . Acesso em: 28 mai. 2020. 
BZUNECK, J. A., BORUCHOVITCH, E.; Adolescence and education: contemporary trends in Brazilian research. In: PAJARES, F.; URDAN, T. (org.). Adolescence and education: international perspectives. Greenwich: Information Age Publishing, 2003.

CAMPELLO, A. V. C.; LINS, L. N. Metodologia de análise e tratamento da evasão e retenção em cursos de graduação de instituições federais de ensino superior. In: XXVIII Encontro Nacional de Engenharia de Produção, 2008, Rio de Janeiro. Anais. Rio de Janeiro, 2008.

CARVALHO, L. F. et al. Aulas interdisciplinares como ferramenta para motivação de ingressantes em engenharia. Revista de Ensino de Engenharia, v. 37, n. 2, p. 46-51, 2018.

CAVENAGUI, A. R. A.; BZUNECK, J. A. A Motivação de alunos adolescentes enquanto desafio na formação do professor. In: IX Congresso Nacional de Educação, 2009, Curitiba. Anais. Curitiba, 2009.

INSTITUTO FEDERAL DE EDUCAÇÃO, CIÊNCIA E TECNOLOGIA DO CEARÁ. Campus Morada Nova. Projeto Pedagógico do Curso de Engenharia Civil. Morada Nova: IFCE, 2015.

Campus Quixadá. Projeto Pedagógico do Curso de Engenharia de Produção Civil. Quixadá: IFCE, 2015a.

Pró-reitora de Ensino. Manual de elaboração de projetos pedagógicos dos cursos do Instituto Federal do Ceará. Fortaleza: IFCE, 2017. IFCE, 2017a.

Campus Quixadá. Projeto Pedagógico do Curso de Engenharia Civil. Quixadá:

Campus Fortaleza. Projeto Pedagógico do Curso de Bacharelado em Engenharia Civil. Fortaleza: IFCE, 2019.

IFCE Institucional. Disponível em: https://ifce.edu.br/acesso-ainformacao/Institucional. Acesso em: 26 mai. 2020.

IFCE em números. Disponível em: http://ifceemnumeros.ifce.edu.br/. Acesso em: 25 mai. 2020a.

MENESES, L. et al. IntroComp: atraindo alunos do ensino médio para uma instigante experiência com a programação. In: 23 Workshop sobre Educação em Informática, 2015, Recife. Anais. Recife, 2015.

NATIONAL RESEARCH COUNCIL. Education for Life and Work: Developing Transferable Knowlwdge and Skills in the 21st Century. J. W. PELLEGRINO, \& M. L. HILTON (org.) Washington: The National Academies Press, 2012.

ORGANISATION FOR ECONOMIC CO-OPERATION AND DEVELOPMENT.

Education at a Glance 2019: OECD Indicators. OECD Publishing: Paris, 2019. 
"Os desafios para formar hoje o engenheiro do amanhã"

OLIVEIRA, V. F. A Engenharia e as novas DCNs: oportunidades para formar mais e melhores engenheiros. Rio de Janeiro: LTC, 2019.

RASK, K. Attrition in STEM fields at a liberal arts college: The importance of grades and pre-collegiate preferences. Economics of Education Review, v. 29, n. 6, p. 892-900, 2010.

SILVA, L. P.; CECÍLIO, S. A mudança no modelo de ensino e de formação na engenharia. Educação em Revista, v. 45. p. 61-80, 2007.

SILVA FILHO, R. L. L. et al. A evasão no ensino superior brasileiro. Cadernos de Pesquisa, v. 37, p. 641-659, 2007.

WATANABE, F. Y. et al. As novas DCNs de engenharia-desafios, oportunidades e proposições. In: IV Congresso de Ensino de Graduação, 2019, São Carlos. Anais. São Carlos, 2019.

YIN, R. K. Estudo de caso: planejamento e métodos. 5. ed. Porto Alegre: Bookman, 2015

\title{
THE NEW DCNS AND ENGINEERING COURSES OF THE FEDERAL INSTITUTE OF EDUCATION, SCIENCE AND TECHNOLOGY OF CEARÁ - BRAZIL
}

\begin{abstract}
The Brazilian Ministry of Education approved the New National Curriculum Guidelines (DCNs) for Undergraduate Engineering Courses through Resolution CNE/CES $n^{\circ}$ 2, in April 2019. The reason for the change is due to the demands of the contemporary work world and the profile of young students. This paper aims to assess the current situation of Pedagogical Projects and the possibilities of inserting new DCNs in engineering courses in the area of Civil Construction at the Federal Institute of Education, Science, and Technology of Ceará (IFCE). The methodological procedures followed the steps for multiple case studies with the development of a checklist (with four categories and sixty-three items), followed by the application in four Pedagogical Projects of engineering courses and analysis and crossing of the results. After the situational diagnosis, it was noticed that the courses fulfill more than $85 \%$ of the items provided for in the new guidelines. However, it is necessary to implement some important points such as the use of active methodologies, the incentive for the participation of junior enterprise, and a faculty development program.
\end{abstract}

Keywords: DCNs. Pedagogical Course Projects. Engineering. IFCE. 